\title{
Dissociating Perceptual Awareness and Postperceptual Processing: The P300 Is Not a Reliable Marker of Somatosensory Target Detection
}

\author{
${ }^{D}$ Pia Schröder, ${ }^{\circ}$ Till Nierhaus, and Felix Blankenburg \\ Neurocomputation and Neuroimaging Unit, Freie Universität Berlin, 14195 Berlin, Germany
}

A central challenge in the study of conscious perception lies in dissociating the neural correlates of perceptual awareness from those reflecting its precursors and consequences. No-report paradigms have been instrumental in this endeavor, demonstrating that the event-related potential P300, recorded from the human scalp, reflects reports rather than awareness. However, these paradigms cannot probe the degree to which stimuli are consciously processed from trial to trial and, thus, leave open the possibility that the P300 is a genuine correlate of conscious access enabling reports. Here, instead of removing report requirements, we took the opposite approach and equated postperceptual task demands across conscious and unconscious trials by orthogonalizing target detection and overt reports in a somatosensory detection task. We used Bayesian model selection to track the transformation from physical to perceptual processing stages in the EEG data of 24 male and female participants and show that the early P50 component scaled with physical stimulus intensity, whereas the N140 component was the first correlate of target detection. The late P300 component was elicited for both perceived and unperceived stimuli and was not substantially modulated by target detection. This was in stark contrast to a control experiment using a classical direct report task, which replicated the P50 and N140 effects but additionally showed a strong effect of target detection in the P300 time range. Our results demonstrate the task dependence of the P300 in the somatosensory modality and show that late cortical potentials dissociate from perceptual awareness even when stimuli are always reported.

Key words: Bayesian model selection; electroencephalography; P300; perceptual awareness; somatosensory; target detection

Significance Statement

The time it takes for sensory information to enter our conscious experience can be an indicator of the neural processing stages that lead to perceptual awareness. However, because many cognitive processes routinely correlate with perception, isolating those signals that uniquely reflect perceptual awareness is not a trivial task. Here, we show that late electroencephalography signals cease to correlate with somatosensory awareness when common task confounds are controlled. Importantly, by balancing report requirements instead of abolishing them, we show that the lack of late effects cannot be explained by a lack of conscious access. Instead, we propose that conscious access occurs earlier, at $\sim 150 \mathrm{~ms}$, supporting the view that early activity in sensory cortices is a neural correlate of conscious perception.

\section{Introduction}

Whether the neural processes giving rise to conscious perception originate in sensory cortices or higher-order regions is a longstanding debate. In the somatosensory modality, the eventrelated potentials (ERPs) N140 over contralateral somatosensory electrodes and P300 over centroparietal electrodes show

Received Nov. 19, 2020; revised Jan. 26, 2021; accepted Mar. 13, 2021

Author contributions: P.S. and F.B. designed research; P.S. and T.N. performed research; P.S. analyzed data;

P.S. wrote the first draft of the paper; P.S., T.N., and F.B. edited the paper; P.S. wrote the paper.

The authors declare no competing financial interests.

Correspondence should be addressed to Pia Schröder at pia.schroeder@fu-berlin.de.

https://doi.org/10.1523/JNEUROSCI.2950-20.2021

Copyright $\odot 2021$ the authors enhanced amplitudes for consciously perceived stimuli (Schubert et al., 2006; Auksztulewicz et al., 2012), whereas very early components, such as the P50, seem to reflect physical stimulus properties (Forschack et al., 2020). These findings are consistent with studies in the visual and auditory modalities that have similarly reported awareness-related negativities at $\sim 200 \mathrm{~ms}$ over modality-specific electrodes and late positivities at $\sim 350$ ms over centroparietal electrodes (Sergent et al., 2005; Eklund et al., 2019).

Despite this agreement, recent years have seen a rigorous debate centering on the question of whether these potentials truly reflect awareness or are instead associated with precursors or consequences of conscious perception (Melloni et al., 2011; Aru et al., 2012; Pitts et al., 2014; Rutiku et al., 2015; Naccache et al., 2016; Cohen et al., 2020; for a recent review, see Förster et al., 2020). 
While early negativities have been suspected to reflect attentional processes rather than awareness (Pitts et al., 2014), the P300 has been found to be sensitive to expectations (Melloni et al., 2011), attention (Koivisto et al., 2006), and the timing of reports (Ye and Lyu, 2019). In a series of studies, Pitts and colleagues have demonstrated that the visual P300, in particular its later subcomponent $\mathrm{P} 3 \mathrm{~b}$, ceases to index awareness when stimuli are conscious but not reported, that is, when they have no behavioral relevance to the task (Pitts et al., 2014; Cohen et al., 2020; Schlossmacher et al., 2020). These findings suggest that the $P 300$ reflects postperceptual processing rather than perceptual awareness per se. However, two questions are still outstanding: First, because no-report paradigms do not directly inquire participants' subjective experience, it remains unclear to what extent the presented stimuli are consciously processed and whether the neural processes that otherwise allow sensory information to become accessible for conscious report can accurately be captured. In this context, it has been criticized that failure to detect a P300 for conscious but task-irrelevant stimuli may be due to increased temporal variability of the elicited components (Boncompte and Cosmelli, 2018), a lack of conscious access, or even a failure to correctly identify conscious stimuli (Mashour et al., 2020). Indeed, studies using more active manipulations of reports have been inconclusive regarding the task dependence of the P300, showing a dependence on reports in vision (Koivisto et al., 2016) but not in audition (Eklund et al., 2019; but see Schlossmacher et al., 2021). This leads to the second question: do findings from visual studies generalize across sensory modalities? So far, studies testing the task dependence of the P300 are scarce in the auditory and completely lacking in the somatosensory modality, prohibiting final conclusions regarding the general relevance of early and late ERP markers of perceptual awareness.

The goal of our study was to address these questions and scrutinize the task dependence of early and late ERP correlates of perceptual awareness in the somatosensory modality. To control for task demands while guaranteeing conscious access, we used a somatosensory-visual matching task that orthogonalized target detection and perceptual reports, leading to equated behavioral relevance, working memory, and attentional capture of detected and undetected stimuli. To track the transformation from physical to perceptual processing stages, we constructed simple behavioral models capturing various task dimensions and evaluated them using time-resolved Bayesian model selection (Stephan et al., 2009). Based on a previous fMRI study (Schröder et al., 2019) and insights from the visual modality, we hypothesized that (1) very early potentials (P50) reflect physical stimulus properties, (2) the somatosensory N140 correlates with target detection independent of task requirements, and (3) the P300 reflects postperceptual processes and only differentiates between hits and misses when reports correlate with detection but not when report requirements are controlled for.

\section{Materials and Methods}

\section{Participants}

Participants were recruited from the student body of the Freie Universität Berlin and from the general public. All participants reported to be healthy with no history of neurologic or psychiatric disorders, were right-handed, and had normal or corrected-to-normal vision. Twentyeight participants completed the main experiment (matching task). Data of 3 participants were excluded because they did not show stable psychometric functions (see Behavior and Fig. 2), and data of another participant were excluded because of strong motion-related artifacts in the EEG recordings leading to the exclusion of $>50 \%$ of trials. Thus, data of 24 participants entered the analyses (19 females, 5 males, age range: 1937 years). Another 23 participants completed a control experiment using a classical detection task (direct report task). From this dataset, data of 1 participant were excluded because of unstable psychometric functions, leaving data of 22 participants that entered the analyses (12 females, 10 males, age range: $21-42$ years). All participants gave written informed consent before the experiment and received a monetary reimbursement or course credits for their participation. The study was approved by the local ethics committee at the Freie Universität Berlin and complied with the Human Subjects Guidelines of the Declaration of Helsinki.

\section{Experimental design}

Matching task. In the main experiment, participants performed a two-alternative forced choice somatosensory detection task on electrical median-nerve stimuli while their EEG was recorded (Fig. 1A). Each trial was preceded by a variable intertrial interval, during which participants had to fixate on a central gray fixation disk. Following the intertrial interval, an electrical target stimulus was delivered at 1 of 10 intensity levels. The intensity levels were individually calibrated to sample the full dynamic range of each participant's psychometric function from $0 \%$ to $100 \%$ detectability (see Stimuli and apparatus). Accordingly, physical stimulus properties, detection probability, and perceptual uncertainty associated with target detection varied from trial to trial. Presentation of the electrical target pulse was accompanied by a simultaneous change in the fixation disk's brightness to either white or dark gray. This change in brightness served as a visual matching cue, which signaled target presence (white) or absence (dark gray). Participants were instructed to compare their somatosensory percept (electrical pulse detected or not detected) to the visual matching cue (signaling target presence or absence) and decide whether the two modalities produced a match (e.g., electrical pulse detected and white matching cue presented) or a mismatch (e.g., electrical pulse detected and dark gray matching cue presented; Fig. 1A, left box). Electrical pulses were presented on every trial and only their intensity levels varied, rendering them subliminal or supraliminal. Target detection for the highest and lowest intensity levels was expected to be relatively stable ( $\sim 0 \%$ detection probability at intensity level 1 and $\sim 100 \%$ detection probability at intensity level 10 ), whereas detection at intermediate intensity levels was expected to fluctuate from trial to trial. In contrast, the two brightness levels of the matching cue were clearly discernible. This ensured that electrical target detection could be directly inferred from the combination of the matching cues presented on each trial and the participant's match or mismatch reports. After a brief delay, in which the fixation disk returned to its original brightness, two color-coded response cues were presented to the left and right of the fixation disk. Participants reported their match/mismatch decisions by making a saccade to the corresponding response cue (the color codes were counterbalanced across participants). Their gaze was evaluated online, and a response was registered as soon as the gaze remained within the response area for $0.2 \mathrm{~s}$, which was signaled to the participant by a brief increase in the selected response cue's size. The next intertrial interval began as soon as a response was logged or once the allotted response time had elapsed. In this case, the fixation disk briefly turned red, signaling a missed trial. The presented matching cues and the specific sides on which the color-coded response cues were presented were counterbalanced across intensity levels, and trials were presented in random order. All participants completed 6 blocks of 200 trials $(\sim 10$ min per block), resulting in 1200 trials per participant. The number of trials per intensity level followed a normal distribution, such that the number of trials at intensity levels near the 50\% detection threshold was maximized (with 192 trials each for the threshold intensity levels 5 and 6 and 48 trials each for the lowest and highest intensity levels 1 and 10). Before the main experiment, participants completed a 30 min training, in which they were required to achieve at least $90 \%$ accuracy on a run with superthreshold stimuli to ensure full understanding of the task and a low error rate in the main experiment.

Direct report task. To assess the influence of task requirements on early and late ERP correlates of somatosensory target detection, we ran a control experiment, in which participants performed a direct report task, comparable to classical detection tasks. The stimulation protocol, visual displays, and all other experimental parameters were identical to the main experiment, and only the task instructions differed to produce hit/ 

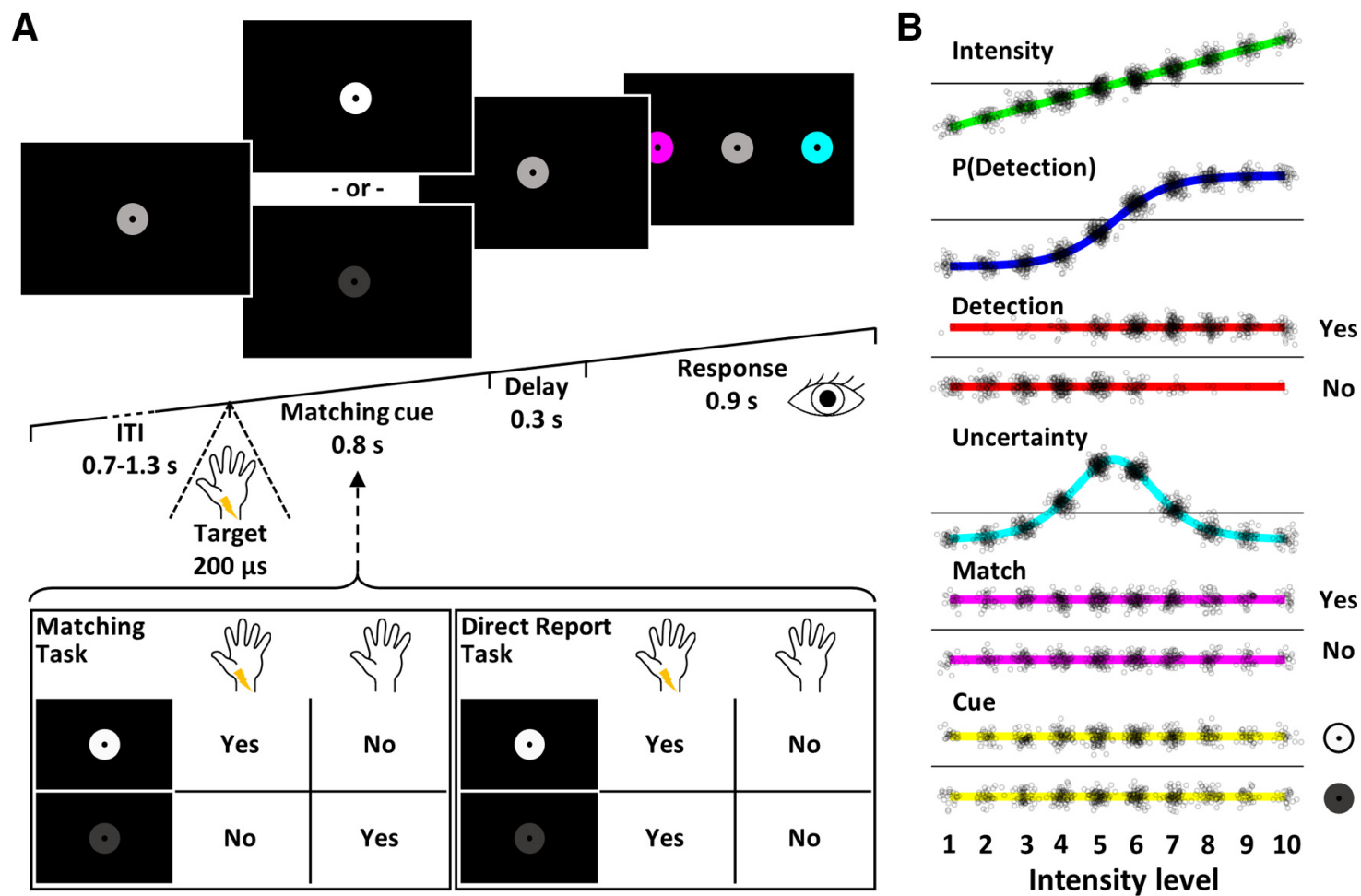

\section{$\begin{array}{lllllllllll}1 & 2 & 3 & 4 & 5 & 6 & 7 & 8 & 9 & 10\end{array}$ Intensity level}

Figure 1. Experimental design. $\boldsymbol{A}$, Trial design. Following a variable intertrial interval, participants received an electrical target pulse at 1 of 10 intensity levels, which they either detected or missed. At the same time, the fixation disk changed its brightness to serve as the visual matching cue, which signaled target presence (white) or target absence (dark gray). In the matching task, participants compared their somatosensory percept to the matching cue and decided whether the two modalities matched or not (left box). In the direct report task, participants ignored the identity of the matching cue and merely decided whether they had detected a target pulse or not (right box). After a brief delay, they reported their decision by making a saccade to one of two peripherally presented, color-coded response cues. The selected cue briefly increased in size, signaling that the response was logged, and the next trial began. $\boldsymbol{B}$, Experimental regressors. EEG responses were modeled with seven different GLMs that were compared using BMS. Each experimental GLM contained an intercept regressor and one of six experimental regressors modeling stimulus intensity, detection probability, target detection (hit vs miss), expected uncertainty, matching reports (match vs mismatch), and matching cues (white vs dark gray). An additional null model contained the intercept regressor only. Small black circles represent individual trials of 1 participant. Please note that although the detection probability regressor is computed from the detection regressor, the two models differ in their behavior within intensity levels: for example, looking at the predictions for trials at intensity level 5, the detection probability model predicts the same activation level for all targets of that intensity level, regardless of whether they were detected or missed, whereas the detection model predicts categorically higher activity for detected than missed trials. Thus, the detection model assumes a nonlinear, all-or-none response for detected stimuli, whereas the detection probability model assumes a graded response. Further note the intensity-biased distribution of trials in the detection regressor, which leads to correlations between models and prohibits classical GLM analysis.

miss reports instead of match/mismatch reports. Participants were similarly presented with electrical target stimuli and concurrent matching cues, but they were informed that the fixation disk's change in brightness merely indicated the timing of a potential target stimulus, with no relevance of the direction of change. Thus, instead of reporting match/mismatch decisions, they directly reported whether they had perceived the target stimulus or not by saccading to the corresponding color-coded response cue (Fig. 1A, right box).

Task comparison. Because of the different report requirements, the two tasks offered different levels of experimental control. In both experiments, the selected intensities ensured an overall detection rate of $\sim 50 \%$, such that detected stimuli were not expected to produce oddball effects. Likewise, because of the randomization, participants could not predict which response cue would be presented on which side, so that they could not prepare a motor response early in the trial in either experiment. However, in the direct report task, target detection directly correlated with overt reports, behavioral relevance, working memory, and attentional capture. In contrast, in the matching task, the use of counterbalanced matching cues resulted in a decorrelation of target detection and overt reports. Importantly, since detected and undetected targets could result in the same overt report, their behavioral relevance and ensuing working memory were equated. Finally, the multimodal nature of the task required participants to split their attentional resources between somatosensory and visual inputs and quickly combine the extracted information into corresponding reports. As a result, signal differences related to poststimulus attentional capture were expected to be minimized. Thus, the contribution of postperceptual processing to the hit versus miss contrast was expected to be considerably attenuated in the matching task compared with the direct report task.

Stimuli and apparatus. Target pulses were generated as analog voltage signals using a waveform generator (DT-9812, Data Translation), converted to direct current monophasic square wave pulses of $200 \mu \mathrm{s} \mathrm{du}-$ ration by a constant current stimulator (DS5, Digitimer), and delivered via adhesive electrodes (GVB-geliMED) attached to the left wrist to stimulate the median nerve. Stimuli were delivered at 10 individually calibrated intensities that were determined from participants' psychometric functions. The psychometric functions were estimated before the experiments using the threshold estimation procedure described by Schröder et al. (2019), which accommodates between-subject variation in detection thresholds and criteria (estimated $1 \%, 50 \%$, and $99 \%$ detection thresholds: matching task: $\mathrm{T} 01=1.88 \pm 0.79 \mathrm{~mA}, \mathrm{~T} 50=2.53 \pm 0.75 \mathrm{~mA}, \mathrm{~T} 99=3.18 \pm 0.91 \mathrm{~mA}$; direct report task: $\mathrm{T} 01=2.38 \pm 0.78 \mathrm{~mA}, \quad \mathrm{~T} 50=2.88 \pm 0.82 \mathrm{~mA}, \mathrm{~T} 99=3.38 \pm$ $0.91 \mathrm{~mA}$ [mean $\pm \mathrm{SD}$ ]). Stimulus presentation was implemented in MATLAB (The MathWorks, RRID:SCR_001622) using the Psychophysics toolbox (Brainard, 1997) (RRID:SCR_002881). For response collection, participants' gaze was measured using an SMI RED-m remote eye tracker $(120 \mathrm{~Hz}$, Sensomotoric Instruments) and SMI's iView X SDK.

EEG recording and preprocessing. EEG data were recorded from 64 electrodes placed according to the extended 10-20 system (ActiveTwo, BioSemi). Four additional electrodes recorded vertical (vEOG) and horizontal (hEOG) eye movements. Preprocessing steps included high-pass filtering at $0.01 \mathrm{~Hz}$ (data of 1 participant in the control experiment were high-pass filtered at $0.5 \mathrm{~Hz}$ to remove excessive sweat artifacts), downsampling from $2048 \mathrm{~Hz}$ to $512 \mathrm{~Hz}$, and rereferencing to the common 
average. Eye blinks were removed from the data using adaptive spatial filtering based on individual blink templates computed from the vEOG (Ille et al., 2002). The continuous data were cut into epochs from -50 to $600 \mathrm{~ms}$ relative to stimulus onset. All epochs were visually inspected for artifacts, and artifactual trials were removed (on average, $5.20 \%$ in the main experiment and $6.48 \%$ in the control experiment). The artifactfree, epoched data were then low-pass filtered at $40 \mathrm{~Hz}$ and baseline corrected using a baseline from -50 to $-5 \mathrm{~ms}$. All preprocessing and data analyses were performed using SPM12 (www.fil.ion.ucl.ac.uk/spm; RRID:SCR_007037) and custom MATLAB scripts. EEGLAB's topoplot function was used to plot topographies (Delorme and Makeig, 2004) (RRID:SCR_007292).

\section{Statistical analysis}

Behavior. Logistic functions were fitted to the behavioral data of each experimental block to obtain continuous models of the underlying psychometric functions (Wichmann and Hill, 2001). For each participant, the estimated slope and threshold parameters were then averaged across blocks to obtain one psychometric function per participant. Estimated detection probabilities $<10 \%$ for intensity level 1 and $>90 \%$ for intensity level 10 were defined as inclusion criteria. The rationale behind these criteria was that minimum and maximum detection probabilities outside these margins would indicate an incomplete sampling of the individual psychometric function (possibly because of changes in detection thresholds, response criteria, or erroneous reports). In the main experiment (matching task), data of 3 participants and in the control experiment (direct report task), data of 1 participant were excluded based on these criteria (see Fig. 2). Differences in mean psychometric functions between tasks, which could be indicative of altered stimulus processing, were tested by running a Bayesian equivalent of the two-sample $t$ test on the estimated $50 \%$ threshold and slope parameters. Differences in reaction times between hits and misses were tested using a Bayesian equivalent of the paired-sample $t$ test (Krekelberg, 2019). For all $t$ tests, Bayes factors in favor of a difference are reported (BF10). To test whether the matching task successfully dissociated target detection from overt reports, Bayesian tests of association (Johnson and Albert, 1999) were performed on these variables for each participant in the matching task and Bayes factors for independence are reported (BF01, i.e., Bayes factors in favor of the null hypothesis). Following the recommendations by Kass and Raftery (1995), we consider $1 \leq \mathrm{BF}<3$ negligible, $3 \leq \mathrm{BF}<20$ positive, $20 \leq \mathrm{BF}<150$ strong, and $150 \leq \mathrm{BF}$ very strong evidence. All descriptive statistics are reported as mean $\pm \mathrm{SD}$, except where otherwise noted.

EEG. Neuronal processing of the somatosensory stimulus was expected to undergo a transformation from physical to perceptual stages and on to the final reports. To track this transformation in the EEG signals and identify the latency at which awareness-related potentials occur, we constructed five GLMs. Each contained an intercept regressor and a trialwise experimental regressor capturing a variable of interest (Fig. 1B): (1) intensity: the stimulus intensity level presented on each trial as a model of physical stimulus properties (linear regressor); (2) P(detection): the individual psychometric functions modeling trialwise detection probability (sigmoidal regressor); (3) detection: categorical target detection which was inferred from match/mismatch reports and matching cues in the main experiment and explicitly reported in the control experiment, modeling a nonlinear response expected to index awareness (binary regressor); (4) uncertainty: the slope of individual psychometric functions modeling the expected uncertainty associated with target detection (inverse U-shaped regressor); and (5) match: matches and mismatches between target detection and visual matching cues, which were explicitly reported in the main experiment and inferred from hit/miss reports and matching cues in the control experiment (binary regressor). Although participants did not engage with the matching cue in the direct report task and did not form match/mismatch reports, we still included the match model (as defined by the alignment of participants' hit/miss reports with the matching cues presented on every trial) in the analysis of both experiments to ensure that any differences in results would be attributable to differences in the data and not to differences in the model spaces. In addition to the five models of interest, we included two further GLMs as control models to validate the approach: (6) cue: a visual
A Matching task

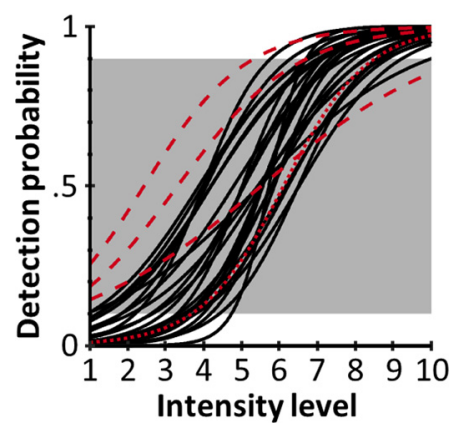

\section{B Direct report task}

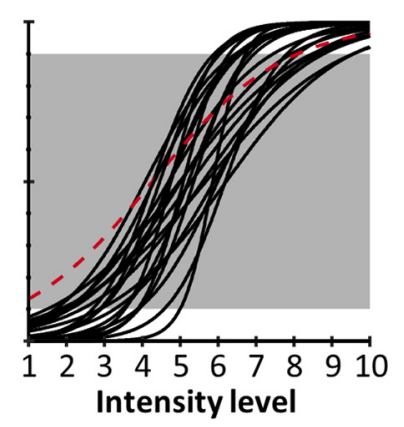

Figure 2. Psychometric functions in the matching task $(\boldsymbol{A})$ and the direct report task $(\boldsymbol{B})$. Black lines indicate the individual block-averaged psychometric functions of participants included in the final samples (matching task: $n=24$; direct report task: $n=22$ ). The psychometric curves are plotted as a function of intensity level, not intensity in $\mathrm{mA}$, to normalize across participants. Red dashed lines indicate participants whose detection probabilities at minimum and maximum intensity levels fell outside the required margin of $<10 \%$ and $>90 \%$ (white background) and were thus excluded from the analyses. The red dotted line indicates 1 participant in the matching task that was excluded because of poor EEG data quality.

control model that was defined by the white or dark gray matching cues to demonstrate specificity of the results to electrodes over somatosensory and visual cortices, respectively (binary regressor); and (7) null: a control model that contained only the intercept regressor to ensure that model selection would correctly dismiss experimental models when no effects were expected (i.e., no effects in the baseline period).

Each of these models captures a unique aspect of the processing stages expected to occur during the tasks, but not all of them are independent. Specifically, because target detection becomes more likely with increasing stimulus intensity, the intensity, detection probability, and detection models are positively correlated. This collinearity complicates classical GLM analysis since corresponding variance in the data cannot be uniquely assigned to any one of the regressors (e.g., the contrast between hits and misses would always be confounded by differences in stimulus intensity). Therefore, we used an alternative approach, Bayesian model selection (BMS) (Stephan et al., 2009), which can capitalize on variance that is explained by each of the models beyond their shared variance. Importantly, the intensity, detection probability, and detection models each make unique predictions: while the intensity and detection probability models both assume a gradual increase of activity with increasing intensity, the intensity model does so in a linear fashion whereas the detection probability model predicts a sigmoidal response profile, with slow changes at low and high intensity levels and a sharp increase near the threshold intensities. Within one intensity level, the detection probability model predicts the same level of activation for all trials of that intensity level, regardless of whether they were detected or not, whereas the detection model predicts categorically higher activity for detected than undetected stimuli, despite being presented at the same intensity level. Accordingly, the detection model assumes an all-or-none response for detected stimuli that is not captured by any of the other models.

To implement the BMS approach, the data from both experiments were analyzed using the same analysis pipeline: the seven GLMs were fitted to participant's trialwise EEG data using the Bayesian estimation scheme as implemented in SPM's spm_vb_glmar.m function. This function approximates the posterior distributions of regression coefficients using variational Bayes (Penny et al., 2003) and provides free energy approximations to the log model evidence (LME), which can be used for model comparison (Penny et al., 2007). To obtain time-resolved estimates of LMEs for each model, electrode, and participant, we fit our GLMs to each time point of each electrode individually (with the autoregressive model order set to zero, effectively reducing the error term to independent and identically distributed Gaussian errors and Gaussian priors for the regression coefficients with mean $\mathrm{w} 0=0$ and variance $\left.\alpha^{-1}=0.005\right)$. All EEG data were $z$-scored across trials before model fitting to obtain data of comparable signal amplitudes. 
To identify which model best explained the EEG data at every time point, the estimated LMEs were then used to perform time-resolved BMS (Stephan et al., 2009). BMS computes exceedance probabilities (EPs) for all models, quantifying the probability that a particular model explains the data better than any of the other models. In addition to enabling model comparison of non-nested models, this approach allows for comparison of models that share variance to different degrees. If models are correlated, their shared variance reduces the relative difference between their LMEs. Accordingly, if correlated and uncorrelated models were included in the comparison individually, the correlated models would be at a disadvantage because they would have to compete against very similar models. At the same time, by assigning equal prior probability to correlated and uncorrelated models, identical portions of variance would be assigned too much prior weight, again resulting in an unfair comparison. BMS offers a simple solution to this problem by presenting the possibility to combine similar models into model families and adjusting the models' prior probabilities accordingly (Penny et al., 2010). The combined models are then assessed on the family level, facilitating fair comparisons. In our case, we combined the intensity, detection probability, and detection models into a model family (which we termed +family because of their positive correlations) and performed BMS on the family level, comparing the +family with the uncertainty, match, cue, and null models. For time points that were best explained by the + family, we further determined which of the individual + family models best explained the data by running the model comparison on the + family models only and weighting the resulting EPs by the + family EP.

To identify time points of interest, we imposed two criteria: (1) the winning model family had to score an EP $\geq 99 \%$ demonstrating a very high probability that the respective model family explained the data better than any of the other model families; and (2) across participants, the $\beta$ estimates of the winning models' experimental regressors had to systematically deviate from zero. Since BMS does not take the directionality of effects across participants into account (LMEs can be high regardless of whether an effect is positive or negative), we added this criterion to ensure that only signals that systematically varied with any of our experimental regressors would be identified. To test this, we extracted $\beta$ estimates of the winning models' experimental regressors and tested these for systematic deviation from zero using a Bayesian equivalent of the one-sample $t$ test (Krekelberg, 2019). Thus, time points of interest were defined as those exceeding both the EP threshold and a $\beta$ evidence threshold of $B F 10 \geq 3$. The null model was exempt of this rule as it did not have an experimental regressor and thus was only required to exceed the EP threshold. When using BMS, it is usually not required to explicitly correct for multiple comparisons, since estimating posterior probabilities does not constitute a statistical test with a binary outcome that would result in false positives (Friston and Penny, 2003). However, when threshold criteria are applied to identify data segments of interest as done here, false positives become a possibility. In this context, it has been noted that thresholding posterior probabilities and labeling the threshold-crossing data segments as "active" is analogous to controlling the false discovery rate in classical inference (Friston and Penny, 2003; Marchini and Presanis, 2004). In our case, by thresholding EPs at $99 \%$, we ensure that, among the data segments surpassing that threshold, at most $1 \%$ may be false positives. Moreover, by using the additional criterion of systematic $\beta$ estimates, unsystematic variation across participants is further prevented from resulting in false positives, such that the true false discovery rate is expected to be even lower.

To visualize the influence of the different task requirements on ERPs elicited by detected and undetected stimuli independent of differences in physical stimulus properties, we extracted subsamples of hit and miss trials that were matched for stimulus intensity. For each participant, we identified all intensity levels that resulted in both hits and misses and randomly sampled trials such that the number of hits and misses was identical within each intensity level (see Fig. $4 A$ ). The subsampled trials were then pooled across intensity levels (main experiment: $225.96 \pm 43.32$ trials per condition; control experiment: $211.23 \pm 47.35$ trials per condition [mean $\pm \mathrm{SD}]$ ) and grand-averaged hit and miss ERPs were plotted for electrodes of interest.

\section{Code and data accessibility}

MATLAB scripts, including all analysis code, are publicly available on Github at: https://github.com/PiaSchroeder/SomatosensoryTargetDetection_ EEG. Preprocessed EEG data and log files holding all relevant trial information can be downloaded from: https://doi.org/10.6084/m9.figshare.13160381.

\section{Results}

\section{Behavior}

Participants detected $53.77 \pm 9.49 \%$ of targets in the matching task and $55.84 \pm 8.99 \%$ in the direct report task. Target detection was most variable on trials with intermediate stimulus intensity levels resulting in characteristic sigmoidal psychometric curves (Fig. 2). The two tasks resulted in similar psychometric curves, as indicated by a lack of evidence for differences in their characteristics (50\% thresholds: matching task T50 $=2.52 \pm 0.79 \mathrm{~mA}$, direct report task $\mathrm{T} 50=2.82 \pm 0.80 \mathrm{~mA}, \mathrm{BF} 10=0.58$; slopes: matching task slope $=7.53 \pm 4.92$, direct report task slope $=$ $8.82 \pm 3.82, \mathrm{BF} 10=0.43$ ). Reaction times were slightly shorter for hits than misses (matching task: hits: $308.97 \pm 32.25 \mathrm{~ms}$, misses: $314.05 \pm 36.52 \mathrm{~ms}, \mathrm{BF} 10=9.27$; direct report task: hits: $313.62 \pm 34.17 \mathrm{~ms}$, misses: $333.89 \pm 42.28 \mathrm{~ms}, \mathrm{BF} 10=568.68$ ). The matching task successfully dissociated target detection from overt reports as suggested by Bayesian tests of association (BF01 $>5$ for all participants).

\section{EEG}

Task dependence of early and late somatosensory ERPs

To test our hypotheses regarding the task dependence of the P50, N140, and P300, we inspected the BMS results in three electrodes of interest: CP4, C6, and CPz. These electrodes were selected based on previous research (Del Cul et al., 2007; Auksztulewicz et al., 2012; Pitts et al., 2014; Cohen et al., 2020), and we confirmed that they captured the components of interest by inspecting grand-averaged topographies of all hit trials across both experiments at relevant time points (P50: $50 \mathrm{~ms}$, N140: $140 \mathrm{~ms}$, P300: 350 ms; Fig. 3). The grand-averaged EEG signals plotted for each intensity level showed the largest deflections for stimuli of high intensity levels and the smallest deflections for stimuli of low intensity levels, suggesting correlation with the +family. This observation was confirmed by BMS: in the matching task, the P50 in contralateral electrode CP4 was best explained by the intensity model, indicating processing of physical stimulus properties at this latency (Fig. 3A; Table 1). The P50 was followed by a centroparietal P100, which was similarly modulated by stimulus intensity but showed a transition to reflect detection probability at $\sim 120 \mathrm{~ms}$. The N140 in electrode C6 was the first to show an effect of target detection at $\sim 150 \mathrm{~ms}$, but this effect was preceded by an effect of physical stimulus intensity, suggesting a transition from physical to perceptual processing stages at this latency. From $\sim 250 \mathrm{~ms}$ onwards, the P300 in electrode $\mathrm{CPz}$ was best explained by the detection probability model (please note that this model assumes no differences between detected and undetected stimuli of the same intensity) and showed little evidence for a late effect of target detection (maximum EP reached by the detection model in this component compared against the intensity and detection probability models: $\mathrm{EP}_{\mathrm{det}}=16.54 \%$, compare Table 1). To get an impression of the spatial extent of this effect, we inspected effect topographies across the scalp for relevant time points and found that the centroparietal electrode cluster constituting the P300 did not show a homogeneous response 


\section{A Matching task}
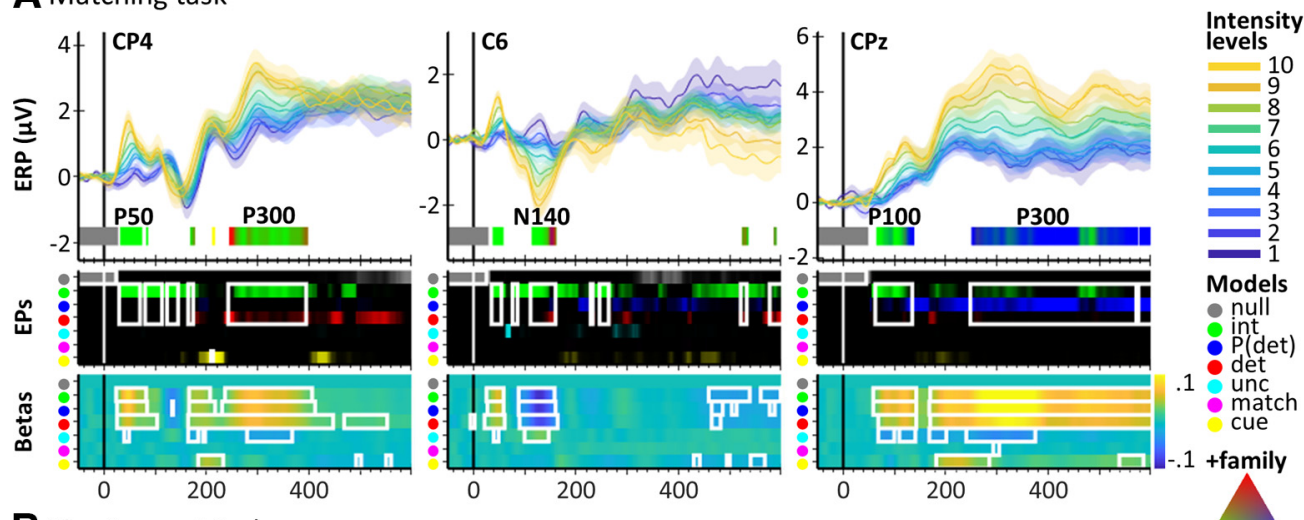

B Direct report task
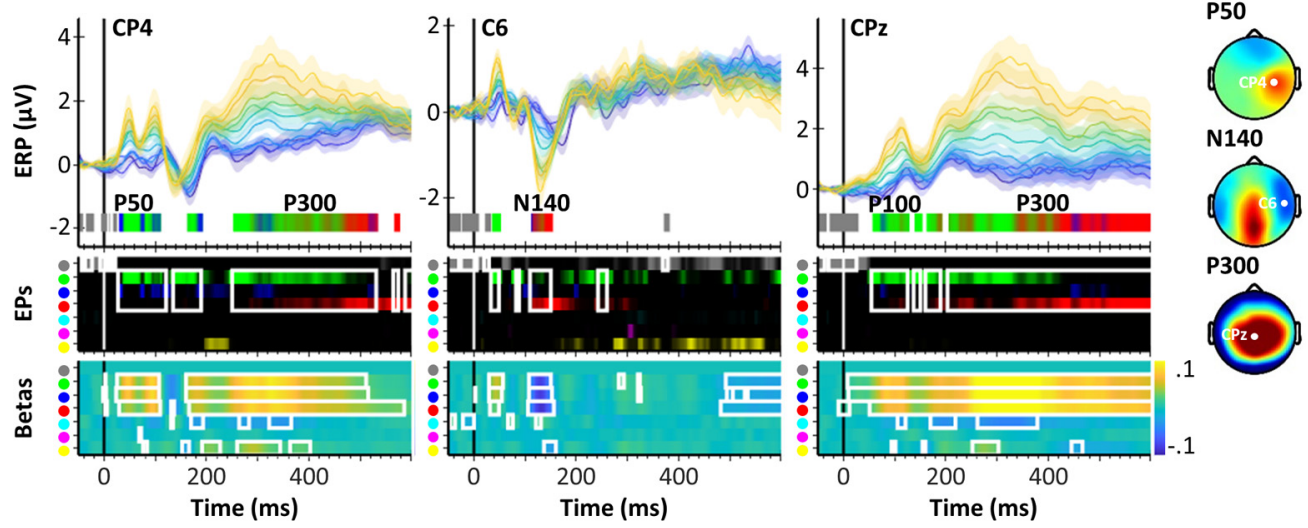

Figure 3. ERPs and BMS results for three electrodes of interest (CP4, $C 6,(\mathrm{CZ}$, marked in grand-averaged Hit topographies on the right) in the matching task $(A)$ and in the direct report task $(\boldsymbol{B})$. For each electrode: Top, Stimulus-locked, grand-averaged ERPs (mean \pm SE) for each intensity level (1-10). Below the ERPs, BMS results are plotted for time points of interest $\left(\mathrm{EP}>=99 \%\right.$ and $\left.\mathrm{BF} 10_{\text {beta }}>=3\right)$ as color bands representing the winning model families. For time points best modeled by the + family (intensity, P(detection), detection), the color represents an RGB value that is composed of the EPs of the three + family models (compare the RGB triangle: corners correspond to $E P=100 \%$, signifying a clear winner of the model comparison within the + family, whereas intermixed colors represent similar EPs for the respective models). Corresponding peak + family model EPs are presented in Table 1. Middle, Unthresholded EP time courses for each model. Bottom, Time courses of group-averaged $\beta$ estimates of each model's experimental regressor (warm colors represent positive $\beta$ estimates; cold colors represent negative $\beta$ estimates). White rectangles represent data segments that exceed the respective thresholds. The results suggest that the P50 was modulated by stimulus intensity in both tasks. The N140 showed a transition from stimulus intensity to target detection in the matching task and a pure effect of target detection in the direct report task. The P300 was strongly task-dependent, showing an effect of detection probability in the matching task and a transition from stimulus intensity to target detection in the direct report task.

Table 1. Peak EPs of + family models as displayed in Figure $3^{a}$

\begin{tabular}{|c|c|c|c|c|c|c|}
\hline & \multicolumn{3}{|l|}{ Matching task } & \multicolumn{3}{|c|}{ Direct report task } \\
\hline & Intensity & $\mathrm{P}$ (detection) & Detection & Intensity & $\mathrm{P}$ (detection) & Detection \\
\hline P50 & 100.00 & $0.00-$ & $0.00-$ & $100.00(56)$ & $1.26 \quad(41)$ & $0.00-$ \\
\hline P100 & 99.92 (102) & $96.95 \quad(135)$ & 17.53 (119) & $100.00(66)$ & 50.91 (76) & 30.75 (111) \\
\hline N140 & 99.97 (123) & 34.12 & 71.96 (156) & 34.61 (133) & 78.73 (111) & 99.87 (145) \\
\hline P300 & 85.64 (482) & 99.99 (393) & $16.54(262)$ & 98.74 (307) & 57.48 & $99.96(578)$ \\
\hline
\end{tabular}

aThe maximum model EPs within time points best explained by the + family in components of interest are presented for the matching task and the direct report task: P50 (40-60 ms, (P4), P100 (60-140 ms, (Pz), N140 (100-160 ms, (6), and P300 (200-600 ms, (Pz). Peak EPs are displayed along with the latency at which they occurred [\% (ms)]. Where a maximum EP occurred repeatedly within a component, the earliest corresponding latency is noted.

across electrodes. Instead, it was dominated by the intensity model in contralateral electrodes and the detection probability model in midline and ipsilateral electrodes (see Fig. 5A).

The control experiment using a direct report task showed similar effects in the early components but marked differences in the late components (Fig. 3B; Table 1). As in the matching task, the P50 and P100 were modulated by stimulus intensity and the N140 showed an effect of target detection. Interestingly, in the direct report task, the detection effect in the N140 dominated the entire component, without a preceding effect of stimulus intensity. However, the most striking difference was seen in the
P300 component. While the early phase of the P300, starting at $\sim 200 \mathrm{~ms}$, was best explained by the intensity model, its later phase, starting at $\sim 350 \mathrm{~ms}$, showed a sustained effect of target detection with an $\mathrm{EP}$ as high as $\mathrm{EP}_{\mathrm{det}}=99.96 \%$ (compare Table 1). Previous studies have shown that the P300 can be subdivided into an earlier, frontocentral P3a and a later, centroparietal P3b (Yamaguchi and Knight, 1991; Polich, 2007). In visual awareness, these subcomponents are assigned different roles: while the $\mathrm{P} 3 \mathrm{a}$ is considered an automatic response that can occur nonconsciously, the P3b is commonly discussed as a candidate marker of awareness (Muller-Gass et al., 2007; Dehaene and Changeux, 2011; Boncompte and Cosmelli, 2018). The transition from physical to perceptual processing stages observed here is, in principle, compatible with this distinction, showing that detection effects occur in the later phase of the P300 (>350 ms, P3b). However, the corresponding effect topographies suggest that the transition from intensity to detection effects occurred similarly across the P300 electrode cluster, with no clear shift from frontocentral to centroparietal electrodes, leaving it somewhat unclear whether the observed effects truly reflect P3a and P3b, respectively (see Fig. 5B).

To further inspect the different detection effects in the two tasks, we plotted grand-averaged subsamples of hit and miss trials that were matched for intensity levels (for details, see Fig. 4A). 


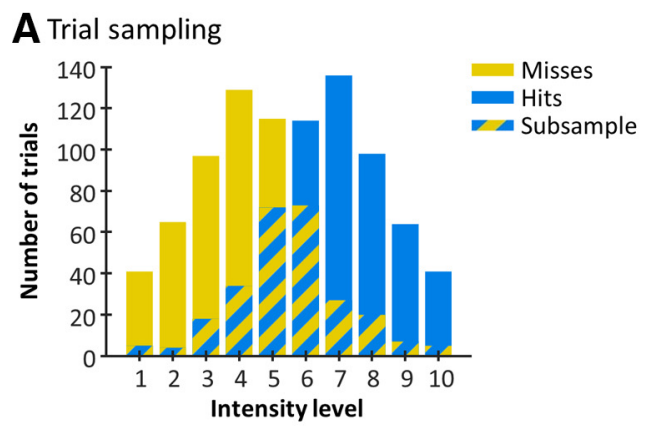

B Matching task
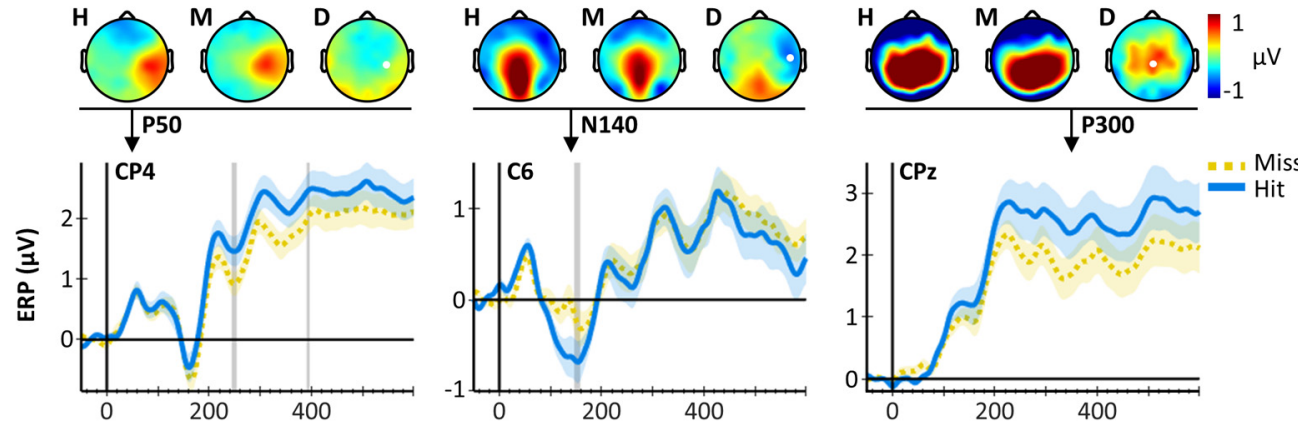

CDirect report task
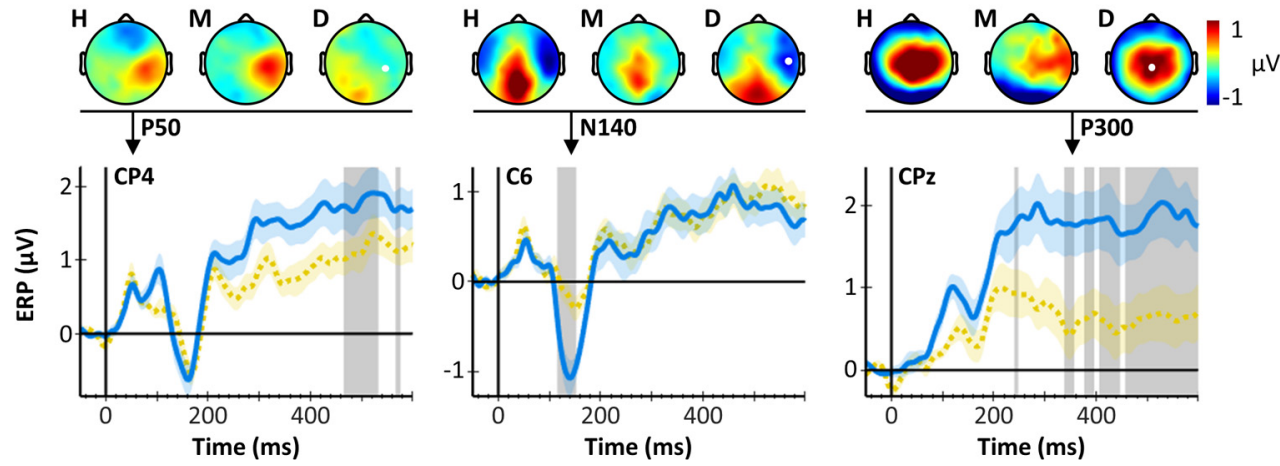

Figure 4. Intensity-matched hit and miss ERPs. A, Trial distributions are shown for one exemplary participant. Lower-intensity levels resulted in more miss trials (yellow), whereas higher-intensity levels resulted in more hit trials (blue). To obtain intensity-matched subsamples of hit and miss trials, for each intensity level, we determined the number of trials obtained per condition and sampled as many trials from the condition with more trials as available for the condition with fewer trials. The subsampled trials (overlap) were then pooled across intensity levels to obtain a hit and a miss pool with identical intensity distributions. $\boldsymbol{B}$, Hit and miss ERPs (mean $\pm \mathrm{SE}$ ) in the matching task and $(\boldsymbol{C})$ in the direct report task. Topographies for hits $(H)$, misses $(M)$, and their difference (D) are displayed for time points of interest (indicated by black arrows). Gray shaded areas represent time points that were best explained by the detection model. The P50 was not modulated by target detection in either task, whereas the N140 exhibits larger amplitudes for hits compared with misses in both tasks. The P300 shows a large difference between hits and misses in the direct report task but not in the matching task.

The resulting ERPs and corresponding scalp topographies showed no difference between hits and misses in the P50 component, further confirming that early EEG signals reflect processing of physical stimulus properties (Fig. 4B,C). The N140, on the other hand, showed a clear amplitude enhancement for hits compared with misses; and this effect was apparent in both experiments, although in the matching task, the detection model only dominated the model comparison in a later time window. In contrast, the P300 clearly reflected the different BMS results in late time windows. In the matching task, hits and misses elicited similar P300 amplitudes, whereas in the direct report task, hits elicited much larger P300 amplitudes than misses, resembling the commonly reported P300 detection effect. Please note that these results represent only a small subset of trials that was largely dominated by near-threshold stimuli (which are susceptible to near-threshold confounds, e.g., the focus of attention and general alertness). For this reason (and to avoid circular analysis), we did not perform any further statistical tests on these data. Nonetheless, this way of looking at the results helps to better understand the evolution of detection effects observed in the BMS results. For example, looking at the intensity-matched hit and miss ERPs in electrode $\mathrm{CPz}$, the late detection effect in the direct report task appears to emerge as early as $200 \mathrm{~ms}$ (compare Fig. 4C). However, the BMS results indicate that the detection effect only started at $\sim 350 \mathrm{~ms}$, whereas earlier time points were explained by stimulus intensity. This suggests that, although target detection in near-threshold trials seems to have had some effect on the signal already at $200 \mathrm{~ms}$, the intensity model still did a better job at explaining the data at this point and only later, at $\sim 350 \mathrm{~ms}$, did the detection model become the most dominant. Similar conclusions may be drawn for the early phase of the N140.

Effects across time and space

To obtain a more global impression of the spatiotemporal evolution of model probabilities, we determined the overall model performances across time as defined by the proportion of electrodes 


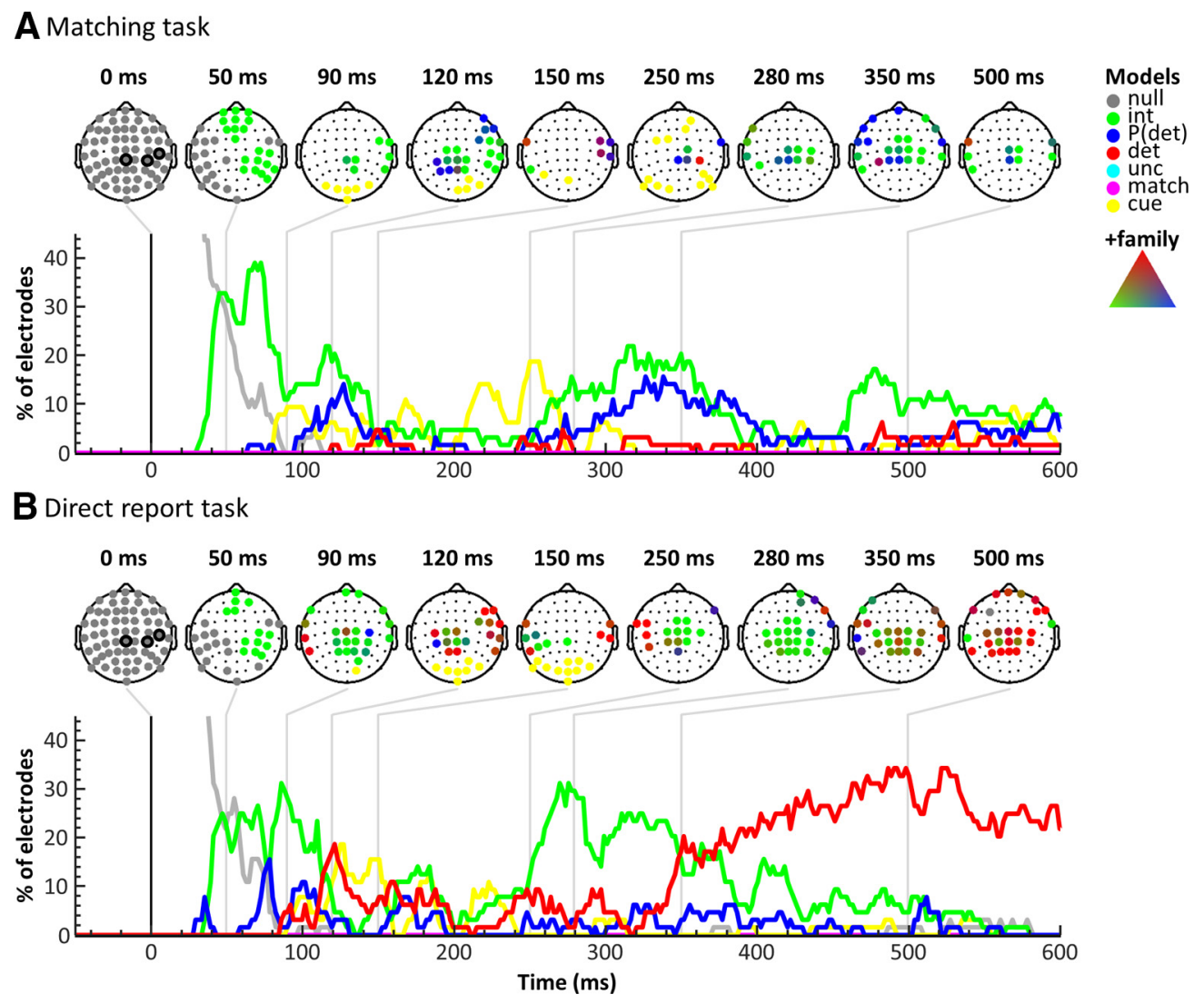

Figure 5. BMS results across electrodes in the matching task $(\boldsymbol{A})$ and the direct report task $(\boldsymbol{B})$. For each task, scalp topographies for time points of interest (top) and model time courses across electrodes (bottom) are displayed. The scalp topographies indicate winning models in electrodes surpassing the threshold criteria using colors as in Figure 3. The circled electrodes at $0 \mathrm{~ms}$ represent electrodes $\mathrm{CP4}$, 6 , and CPz. Model time courses are plotted as the proportion of electrodes showing above-threshold effects over time. The results suggest a striking reduction of target detection effects (red) in the matching task compared with the direct report task, especially in late time windows $>350 \mathrm{~ms}$.

showing above-threshold effects for each model (Fig. 5). As expected, activity in the baseline period was best explained by the null model, validating the specificity of our analysis approach. The intensity model showed similar effects in both tasks: the first clear evidence of somatosensory stimulus processing emerged at $\sim 50 \mathrm{~ms}$, consistent with the P50 response. The BMS topographies at this time point showed a widespread intensity effect in contralateral somatosensory and frontal electrodes, which rotated slightly throughout the following $\sim 100 \mathrm{~ms}$ to include more centroparietal electrodes. Then, starting at $\sim 250 \mathrm{~ms}$, a second strong intensity effect occurred, encompassing primarily centroparietal electrodes. The detection probability model first explained the data in the matching task at $\sim 120 \mathrm{~ms}$ and showed an additional late effect starting at $\sim 250 \mathrm{~ms}$ that paralleled the late intensity effect. Both of these effects showed a centroparietal topography, but the intensity effect occurred primarily in contralateral electrodes, whereas the detection probability effect was mostly found in slightly more posterior midline and ipsilateral electrodes. In the direct report task, the detection probability model showed several smaller peaks with similar topographies as the early intensity effect, but across time and electrodes the detection probability model did not explain the data well in this experiment. The detection model showed the most striking difference between the two experiments. In the matching task, the earliest effect of target detection was observed at the N140 latency, and this effect was confined to only two electrodes (C6 and FC6) and a brief period of time. Likewise, detection effects in later time windows were sparse with only very brief effects in isolated electrodes. In contrast, in the direct report task, the earliest detection effect occurred at $\sim 90 \mathrm{~ms}$ in ipsilateral temporal electrodes, followed by a more widespread detection effect in contralateral central and frontal electrodes at $\sim 120 \mathrm{~ms}$ (including the N140) and yet another effect at $\sim 250 \mathrm{~ms}$ in ipsilateral central electrodes. Finally, from $\sim 350$ ms onwards, a large centroparietal electrode cluster reflected target detection throughout the rest of the time window. These results suggest a striking difference between the two tasks, with a widespread centroparietal detection effect at $\sim 350 \mathrm{~ms}$ in the direct report task that was virtually absent in the matching task. Surprisingly, neither the uncertainty nor the match model scored high EPs in either task, and this lack of effects was unaltered by inspecting later time points or data segments that had not passed the threshold criteria. However, we found effects of the matching cue in both tasks, starting at $\sim 90 \mathrm{~ms}$ in occipital electrodes. In the matching task, this effect lasted slightly longer (until $\sim 300 \mathrm{~ms}$ ) and encompassed frontal and parietal electrodes. This difference was unsurprising given that further processing of the matching cue was vital to the matching task but not to the direct report task.

\section{Discussion}

In this study, we scrutinized the relevance of early and late ERP components as markers of somatosensory awareness. Both in a revised detection task that used a matching procedure to control for report requirements and a control experiment with direct reports, the somatosensory P50 component was modulated by physical stimulus intensity, confirming Hypothesis 1 . The N140 was modulated by target detection in both tasks, although in the 
matching task, this modulation occurred later and was preceded by an effect of stimulus intensity, such that Hypothesis 2 was only partly confirmed. The P300 was clearly task-dependent, showing a widespread detection effect in the direct report task that was largely absent in the matching task, when report requirements were controlled for. These results confirmed Hypothesis 3 and suggest that the P300 is not a reliable marker of somatosensory awareness but reflects postperceptual processing.

The intensity effect observed in the P50 component replicates a recent ERP study that has similarly shown scaling of the P50 amplitude with stimulus strength (Forschack et al., 2020). More generally, our finding concurs with research showing that early stimulus processing in primary somatosensory cortex does not differentiate between detected and undetected stimuli but reflects physical stimulus properties in both humans (Schröder et al., 2019) and macaques (de Lafuente and Romo, 2005, 2006). We thus add to the growing consensus that very early sensory potentials index preconscious processing (Railo et al., 2011; Wühle et al., 2011; Rutiku et al., 2016; Forschack et al., 2020) and support the notion that initial feedforward processing in early sensory regions is not sufficient for conscious perception (Lamme, 2006).

The degree to which early negativities are task-dependent is not well understood. Here, the somatosensory N140 component was modulated by target detection even when postperceptual requirements were controlled for, corroborating its relevance for somatosensory awareness (Schubert et al., 2006; Zhang and Ding, 2010; Auksztulewicz et al., 2012; Auksztulewicz and Blankenburg, 2013; Al et al., 2020; Forschack et al., 2020). However, the BMS analysis revealed that, in the matching task, the N140 was first modulated by stimulus intensity before transitioning to an effect of target detection. Conversely, the N140 in the direct report task was exclusively modulated by target detection, suggesting that the different task demands had a considerable influence on the component's timing and distribution. Indeed, the N140 has previously been reported to be modulated by exogenous attention (Mena et al., 2020). The matching task enforced a reduction of poststimulus attentional engagement compared with the direct report task, which may have resulted in the reduced detection effect. This finding could have important implications for the interpretation of awareness-related negativities in general. Similar to the visual awareness negativity (Koivisto and Grassini, 2016) and auditory awareness negativity (Giani et al., 2015), the N140 has been suggested to result from recurrent interactions between sensory cortices (Auksztulewicz et al., 2012). Accordingly, these potentials have been taken as evidence for the Recurrent Processing Theory of conscious perception, which assumes that perceptual awareness emerges as soon as initial feedforward activity in sensory cortices is consolidated by re-entrant feedback (Lamme, 2006). Indeed, a number of studies have demonstrated detection-related feedback signals from secondary to primary somatosensory cortex in humans (Jones et al., 2007; Auksztulewicz et al., 2012; Auksztulewicz and Blankenburg, 2013), mice (Kwon et al., 2016; Yang et al., 2016), and macaques (Cauller and Kulics, 1991), supporting the role of local recurrent processing for somatosensory awareness. Our results are in principle compatible with Recurrent Processing Theory, but they also suggest that at least part of the awareness effects routinely reported at the N140 latency may be due to uncontrolled processes; and more direct manipulations of attentional allocation are necessary to elucidate this issue.
The P300 has previously been demonstrated to depend on report requirements in visual tasks (Pitts et al., 2014; Cohen et al., 2020; Schlossmacher et al., 2020), and we show that the same is true in the somatosensory modality. In opposition to early negativities, the P300 has been taken as evidence in support of the Global Neuronal Workspace Theory. Global Neuronal Workspace Theory assumes that any conscious percept presupposes a nonlinear "ignition" of global workspace neurons that are distributed across a network of brain regions, primarily in frontal and parietal cortices (Dehaene and Naccache, 2001). Given that several studies have now shown that the P300, often considered a hallmark of ignition (Dehaene and Changeux, 2011), vanishes in no-report paradigms, some researchers have suggested that early activity may reflect general "information accessibility," whereas late activity reflects proper "conscious access," which might be lacking when perception is not reported (Mashour et al., 2020). However, this explanation is not compatible with our results, since we show that a P300 can be elicited regardless of conscious access, further corroborating its postperceptual nature. Alternatively, one might argue that, because attentional resources had to be divided between the somatosensory and visual inputs in the matching task, the attention allocated to the electrical stimuli was not sufficient for them to truly become conscious, such that they remained "preconscious" (Dehaene et al., 2006). However, both the somatosensory and visual stimuli were highly relevant to the task, making it unlikely that attention was withdrawn to the point that the electrical pulses were prevented from becoming conscious. Moreover, we did not find any differences in psychometric functions between the experiments, which one might expect if they differed in their levels of conscious processing. Thus, although we cannot entirely exclude the possibility that there were subtle differences in perceptual experience between the two tasks, the task requirements and behavioral data lead us to believe that the electrical targets were indeed consciously perceived in both tasks, especially given that many of them were presented at superthreshold intensities. Our findings concur with results from fMRI studies showing that frontoparietal network activity does not correlate with conscious access when reports are controlled for (Frässle et al., 2014; Farooqui and Manly, 2018; Schröder et al., 2019). The apparent absence of any sign of ignition in these studies seems to suggest that such widespread, nonlinear activity may not be as relevant for conscious perception as postulated by Global Neuronal Workspace Theory. However, we must note that such findings do not necessarily invalidate the role of frontal or parietal regions for conscious perception, since univariate EEG and fMRI analyses are limited to studying the collective activity of large populations of neurons. It may well be that smaller assemblies or patterns of neurons in these regions correlate with awareness even when task demands are controlled for (see e.g., Kapoor et al., 2020). Nonetheless, research such as ours certainly suggests that the correlates of perceptual awareness are much more subtle than often claimed and that findings from studies showing large signal divergences for conscious stimuli without properly controlling for task demands cannot unreservedly be taken as evidence for any theory of conscious perception (Pitts et al., 2014; Tsuchiya et al., 2015).

Given the ubiquity of the P300 in studies on perceptual awareness, it is worth considering which cognitive variables are its most likely generators and should therefore be most rigorously controlled. In a recent review, Verleger (2020) argues that stimulus-response-link reactivation, memory storage, and closure of cognitive epochs are the most relevant candidates. In 
both our tasks, timing cues indicated the exact moment of a potential stimulus delivery and, thus, controlled for closure of cognitive epochs. However, the two tasks clearly differed in their control of stimulus-response-link reactivation and memory storage, which may have caused the different results. In the series of no-report experiments conducted by Pitts and colleagues (Pitts et al., 2014; Cohen et al., 2020; Schlossmacher et al., 2020), the stimuli of interest were not relevant to the task, such that none of the processes in question were initiated upon stimulus perception and no P300 was elicited. In contrast, Sanchez et al. (2020) used a go/no-go paradigm with response reversals and were able to decode target detection from late MEG activity. However, because of unpredictable stimulus timing and a response delay, their task was not controlled for closure or working memory effects, which may have caused the late effects. Koivisto et al. (2016) used a similar task but incorporated timing cues and speeded responses, leading to better control of closure and memory effects, and they found a modulation of the P300 by reports. However, the same task in the auditory modality did not reveal any effect of reports on the P300 (Eklund et al., 2019). Whether this discrepancy reflects genuine differences between modalities or results from residual task confounds remains to be tested.

Although the P300 in our study was clearly affected by report requirements, it did not correlate with reports in the matching task. Indeed, the match and uncertainty models did not explain any segments of the EEG data in either task. Possibly, the anatomic locations of regions previously found to show such effects (on the medial wall inside the longitudinal fissure or folded deeply into the cortex) (Schröder et al., 2019) prevented strong influences on the overall EEG signal, leading the respective effects to go unnoticed or, alternatively, signals related to uncertainty and matching reports may not have been time-locked to the stimuli. Whatever the reason, since both perceptual uncertainty and overt reports were controlled for in the matching task, the observed detection effects were free of these confounds (for a more extensive discussion of this point, see Schröder et al., 2019). Instead of reflecting overt reports, the P300 in the matching task was best explained by the intensity and detection probability models, potentially suggesting that neither of these models perfectly accounted for the trial-to-trial response variations in this component. A potential interpretation may be that these models captured variability related to the perceptual quality of the stimuli, which is expected to covary with stimulus intensity, at least for detected targets. The goal of our study prohibited subjective awareness ratings, but a previous study has indeed demonstrated that the N140 and P300 amplitudes correlate with graded awareness levels (Auksztulewicz and Blankenburg, 2013). Assuming a graded nature of awareness (Overgaard et al., 2006) might also explain why the effects of categorical target detection were so limited in the matching task. Interestingly, in our fMRI study using the same task (Schröder et al., 2019), effects of stimulus intensity and detection probability were limited to areas in primary and secondary somatosensory cortex. Whether the late signals observed here also originate from these regions remains to be established.

In conclusion, our study demonstrates that conscious access of somatosensory information is not reflected in widespread cortical activation as indexed by the P300 but instead seems to manifest in earlier, locally restricted activity over somatosensory regions. The generally sparse effects of categorical target detection in our study speak against global, nonlinear effects of conscious somatosensory perception. Indeed, focusing on large signal divergences for perceived versus unperceived stimuli may be misleading as they seem to emphasize postperceptual processing rather than perceptual awareness. Our study further stresses the need for carefully controlled experimental paradigms to be able to unequivocally attribute observed effects to stimulus awareness and effectively further our understanding of the neural processes supporting the emergence of conscious perception.

\section{References}

Al E, Iliopoulos F, Forschack N, Nierhaus T, Grund M, Motyka P, Gaebler M, Nikulin VV, Villringer A (2020) Heart-brain interactions shape somatosensory perception and evoked potentials. Proc Natl Acad Sci USA 117:10575-10584.

Aru J, Bachmann T, Singer W, Melloni L (2012) Distilling the neural correlates of consciousness. Neurosci Biobehav Rev 36:737-746.

Auksztulewicz R, Blankenburg F (2013) Subjective rating of weak tactile stimuli is parametrically encoded in event-related potentials. J Neurosci 33:11878-11887.

Auksztulewicz R, Spitzer B, Blankenburg F (2012) Recurrent neural processing and somatosensory awareness. J Neurosci 32:799-805.

Boncompte G, Cosmelli D (2018) Neural correlates of conscious motion perception. Front Hum Neurosci 12:355.

Brainard DH (1997) The Psychophysics Toolbox. Spat Vis 10:433-436.

Cauller LJ, Kulics AT (1991) The neural basis of the behaviorally relevant N1 component of the somatosensory-evoked potential in SI cortex of awake monkeys: evidence that backward cortical projections signal conscious touch sensation. Exp Brain Res 84:607-619.

Cohen MA, Ortego K, Kyroudis A, Pitts M (2020) Distinguishing the neural correlates of perceptual awareness and postperceptual processing. J Neurosci 40:4925-4935.

de Lafuente V, Romo R (2005) Neuronal correlates of subjective sensory experience. Nat Neurosci 8:1698-1703.

de Lafuente V, Romo R (2006) Neural correlate of subjective sensory experience gradually builds up across cortical areas. Proc Natl Acad Sci USA 103:14266-14271.

Dehaene S, Changeux JP (2011) Experimental and theoretical approaches to conscious processing. Neuron 70:200-227.

Dehaene S, Naccache L (2001) Towards a cognitive neuroscience of consciousness: basic evidence and a workspace framework. Cognition 79: $1-37$.

Dehaene S, Changeux JP, Naccache L, Sackur J, Sergent C (2006) Conscious, preconscious, and subliminal processing: a testable taxonomy. Trends Cogn Sci 10:204-211.

Del Cul A, Baillet S, Dehaene S (2007) Brain dynamics underlying the nonlinear threshold for access to consciousness. PLoS Biol 5:e260.

Delorme A, Makeig S (2004) EEGLAB: an open source toolbox for analysis of single-trial EEG dynamics including independent component analysis. J Neurosci Methods 134:9-21.

Eklund R, Gerdfeldter B, Wiens S (2019) Effects of a manual response requirement on early and late correlates of auditory awareness. Front Psychol 10:2083.

Farooqui AA, Manly T (2018) When attended and conscious perception deactivates fronto-parietal regions. Cortex 107:166-179.

Forschack N, Nierhaus T, Müller MM, Villringer A (2020) Dissociable neural correlates of stimulation intensity and detection in somatosensation. Neuroimage 217:116908.

Förster J, Koivisto M, Revonsuo A (2020) ERP and MEG correlates of visual consciousness: the second decade. Conscious Cogn 80:102917.

Frässle S, Sommer J, Jansen A, Naber M, Einhäuser W (2014) Binocular rivalry: frontal activity relates to introspection and action but not to perception. J Neurosci 34:1738-1747.

Friston KJ, Penny W (2003) Posterior probability maps and SPMs. Neuroimage 19:1240-1249.

Giani AS, Belardinelli P, Ortiz E, Kleiner M, Noppeney U (2015) Detecting tones in complex auditory scenes. Neuroimage 122:203-213.

Ille N, Berg P, Scherg M (2002) Artifact correction of the ongoing EEG using spatial filters based on artifact and brain signal topographies. J Clin Neurophysiol 19:113-124.

Johnson VE, Albert J (1999) Ordinal data modeling. New York: Springer.

Jones SR, Pritchett DL, Stufflebeam SM, Hämäläinen M, Moore CI, Hamalainen M, Moore CI (2007) Neural correlates of tactile detection: a 
combined magnetoencephalography and biophysically based computational modeling study. J Neurosci 27:10751-10764.

Kapoor V, Dwarakanath A, Safavi S, Werner J, Besserve M, Panagiotaropoulos T, Logothetis N (2020) Decoding the contents of consciousness from prefrontal ensembles. bioRxiv. doi: 10.1101/ 2020.01.28.921841.

Kass RE, Raftery AE (1995) Bayes factors. J Am Stat Assoc 90:773-795.

Koivisto M, Grassini S (2016) Neural processing around $200 \mathrm{~ms}$ after stimulus-onset correlates with subjective visual awareness. Neuropsychologia 84:235-243.

Koivisto M, Revonsuo A, Lehtonen M (2006) Independence of visual awareness from the scope of attention: an electrophysiological study. Cereb Cortex 16:415-424.

Koivisto M, Salminen-Vaparanta N, Grassini S, Revonsuo A (2016) Subjective visual awareness emerges prior to P3. Eur J Neurosci 43:16011611.

Krekelberg B (2019) bayesFactor. Available at https:/github.com/klabhub/ bayesFactor.

Kwon SE, Yang H, Minamisawa G, O'Connor DH (2016) Sensory and decision-related activity propagate in a cortical feedback loop during touch perception. Nat Neurosci 19:1243-1249.

Lamme VA (2006) Towards a true neural stance on consciousness. Trends Cogn Sci 10:494-501.

Marchini J, Presanis A (2004) Comparing methods of analyzing fMRI statistical parametric maps. Neuroimage 22:1203-1213.

Mashour GA, Roelfsema P, Changeux JP, Dehaene S (2020) Conscious processing and the global neuronal workspace hypothesis. Neuron 105:776798.

Melloni L, Schwiedrzik CM, Müller N, Rodriguez E, Singer W (2011) Expectations change the signatures and timing of electrophysiological correlates of perceptual awareness. J Neurosci 31:1386-1396.

Mena CI, Lang K, Gherri E (2020) Electrophysiological correlates of attentional selection in tactile search tasks: the impact of singleton distractors on target selection. Psychophysiology 57:1-12.

Muller-Gass A, Macdonald M, Schröger E, Sculthorpe L, Campbell K (2007) Evidence for the auditory P3a reflecting an automatic process: elicitation during highly-focused continuous visual attention. Brain Res 1170:71-78.

Naccache L, Marti S, Sitt JD, Trübutschek D, Berkovitch L (2016) Why the P3b is still a plausible correlate of conscious access? A commentary on Silverstein et al., 2015. Cortex 85:126-128.

Overgaard M, Rote J, Mouridsen K, Ramsøy TZ (2006) Is conscious perception gradual or dichotomous? A comparison of report methodologies during a visual task. Conscious Cogn 15:700-708

Penny W, Kiebel S, Friston K (2003) Variational Bayesian inference for fMRI time series. Neuroimage 19:727-741.

Penny W, Flandin G, Trujillo-Barreto N (2007) Bayesian comparison of spatially regularised general linear models. Hum Brain Mapp 28:275-293.

Penny W, Stephan KE, Daunizeau J, Rosa MJ, Friston K, Schofield TM, Leff AP (2010) Comparing families of dynamic causal models. PLoS Comput Biol 6:e1000709.

Pitts MA, Metzler S, Hillyard SA (2014) Isolating neural correlates of conscious perception from neural correlates of reporting one's perception. Front Psychol 5:1078.
Polich J (2007) Updating P300: an integrative theory of P3a and P3b. Clin Neurophysiol 118:2128-2148.

Railo H, Koivisto M, Revonsuo A (2011) Tracking the processes behind conscious perception: a review of event-related potential correlates of visual consciousness. Conscious Cogn 20:972-983.

Rutiku R, Martin M, Bachmann T, Aru J (2015) Does the P300 reflect conscious perception or its consequences? Neuroscience 298:180-189.

Rutiku R, Aru J, Bachmann T (2016) General markers of conscious visual perception and their timing. Front Hum Neurosci 10:23.

Sanchez G, Hartmann T, Fuscà M, Demarchi G, Weisz N (2020) Decoding across sensory modalities reveals common supramodal signatures of conscious perception. Proc Natl Acad Sci USA 117:7437-7446.

Schlossmacher I, Dellert T, Pitts M, Bruchmann M, Straube T (2020) Differential effects of awareness and task relevance on early and late ERPs in a no-report visual oddball paradigm. J Neurosci 40:2906-2913.

Schlossmacher I, Dellert T, Bruchmann M, Straube T (2021) Dissociating neural correlates of consciousness and task relevance during auditory processing. Neuroimage 228:117712.

Schröder P, Schmidt TT, Blankenburg F (2019) Neural basis of somatosensory target detection independent of uncertainty, relevance, and reports. Elife 8:e43410.

Schubert R, Blankenburg F, Lemm S, Villringer A, Curio G (2006) Now you feel it-now you don't: ERP correlates of somatosensory awareness. Psychophysiology 43:31-40.

Sergent C, Baillet S, Dehaene S (2005) Timing of the brain events underlying access to consciousness during the attentional blink. Nat Neurosci 8:1391-1400.

Stephan KE, Penny W, Daunizeau J, Moran RJ, Friston K (2009) Bayesian model selection for group studies. Neuroimage 46:1004-1017.

Tsuchiya N, Wilke M, Frässle S, Lamme VA (2015) No-report paradigms: extracting the true neural correlates of consciousness. Trends Cogn Sci 19:757-770.

Verleger R (2020) Effects of relevance and response frequency on P3b amplitudes: review of findings and comparison of hypotheses about the process reflected by P3b. Psychophysiology 57:e13542.

Wichmann FA, Hill NJ (2001) The psychometric function: I. Fitting, sampling, and goodness of fit. Percept Psychophys 63:1293-1313.

Wühle A, Preissl H, Braun C (2011) Cortical processing of near-threshold tactile stimuli in a paired-stimulus paradigm: an MEG study. Eur J Neurosci 34:641-651.

Yamaguchi S, Knight RT (1991) P300 generation by novel somatosensory stimuli. Electroencephalogr Clin Neurophysiol 78:50-55.

Yang H, Kwon SE, Severson KS, O'Connor DH (2016) Origins of choicerelated activity in mouse somatosensory cortex. Nat Neurosci 19:127134.

Ye M, Lyu Y (2019) Later positivity reflects post-perceptual processes: evidence from immediate detection and delayed detection tasks. Front Psychol 10:82.

Zhang Y, Ding M (2010) Detection of a weak somatosensory stimulus: role of the prestimulus mu rhythm and its top-down modulation. J Cogn Neurosci 22:307-322. 